\title{
Dose-dependent effect of capsaicin on endurance capacity in rats
}

\author{
Tae-Woong Oh* and Fukio Ohta \\ Department of Sports Sciences, School of Human Sciences, Waseda University, Japan
}

(Received 6 August 2002 - Revised 28 March 2003 - Accepted 17 April 2003)

\begin{abstract}
The present study was designed to determine the effects of various levels of capsaicin (CAP) on endurance capacity in forty-nine male Sprague-Dawley rats, aged 4 weeks, which were assigned to four groups. Rats were given orally either control $(0)$ or $6,10 \mathrm{or} 15 \mathrm{mg}$ $\mathrm{CAP} / \mathrm{kg}$ body weight $2 \mathrm{~h}$ before exercise by stomach intubations using a round-ended needle. The rats in each group were divided into two subgroups for resting or swimming exercise. Swimming exercise was performed with a weight corresponding to $3 \%$ body weight attached to the tail, and endurance capacity was evaluated by swimming time to exhaustion. The highest dose $(15 \mathrm{mg} / \mathrm{kg})$ of CAP significantly $(P<0.05)$ increased endurance performance time and caused plasma non-esterified fatty acid to rise significantly by about 1.4 -fold compared with that of non-CAP treated rats at exhaustion $(P<0.05)$. The highest dose of CAP had no effect on liver and gastrocnemius muscle glycogen (white and red) in resting rats, but significantly increased glycogen contents of both muscles at exhaustion $(P<0 \cdot 05)$. At rest, plasma noradrenalin levels of the rats receiving the highest dose were greater than that of non-CAP-treated rats and remained greater until exhaustion. The effects of CAP on endurance capacity have received little attention and have conveyed conflicting impressions. Kim et al. (1997) failed to show the maximal effect of $10 \mathrm{mg} / \mathrm{kg}$ doses of CAP on swimming endurance time in mice. The influences of various doses of CAP on endurance capacity were still unclear. Matsuo et al. (1996) reported that the intake of CAP have little sparing effect on glycogen in the liver and soleus muscles at rest and during exercise in rats previously fed a CAP-containing diet ad libitum for 1 week. Our present results suggest more than the highest dose of CAP $(15 \mathrm{mg} / \mathrm{kg})$ can cause the increase of endurance capacity, which might be induced through the sparing of muscle glycogen and the rise of non-esterfied fatty acids following the increase of circulating catecholamine.
\end{abstract}

Capsaicin: Catecholamine: Swimming: Endurance capacity: Non-esterified fatty acid

Capsaicin (CAP) is a pungent component of hot red peppers, which are widely used as an important spice for enhancing the palatability of food, and also utilized as a medicine for developing counter-irritation (Wachtel, 1999).

Kim et al. (1997) demonstrated that oral administration of CAP resulted in increased endurance time during prolonged work. These increases were associated with enhanced lipolysis and sparing of stored glycogen, which results in delaying complete glycogen depletion by an increase in circulating catecholamine. The enhanced availability of non-esterfied fatty acids (NEFA) was thought to cause greater fat metabolism in the active muscle, which in turn inhibited carbohydrate metabolism and resulted in increased exercise capacity. We have recently demonstrated that CAP added to a high-fat diet $(0 \cdot 14 \mathrm{~g} \mathrm{CAP} / \mathrm{kg})$ raised serum NEFA concentrations in rats, which possibly led to an increase in fat utilization as a energy source (Oh, 2001). Kawada et al. (1986) reported that CAP administration transiently induced a higher RQ and then markedly lowered it to about 0.75 .

The importance of the amount of glycogen stored in working muscles for the endurance capacity has been described (Leighton \& Foot, 1995). Increased fat utilization during endurance exercise enables athletes to improve endurance capacity (Azevedo et al. 1998). Therefore, the increase of fatty acid utilization during exercise is supposed to improve endurance capacity.

There have been very few studies of exercise and different doses of CAP. Kim et al. (1997) failed to show any effects of $3-15 \mathrm{mg} \mathrm{CAP} / \mathrm{kg}$ on endurance time during swimming exercise tests. However, there was no CAP effect at $15 \mathrm{mg} / \mathrm{kg}$. The dose relationships of CAP and metabolism during exercise have not been studied in detail; none of the few studies measured plasma hormones. By examining the endurance time, metabolic and hormonal responses to various CAP doses, one could gain a deeper understanding of how the effects of CAP are mediated. In the present study, we investigated the effects of light, moderate and heavy doses of CAP on endurance capacity and glycogenolysis by exhaustive swimming exercise in rats.

\section{Materials and methods \\ Animal care and experimental design}

Forty-nine male Sprague-Dawley rats, aged 4 weeks and weighing 75-85g, were purchased from Japan Charles 
River Co., Ltd (Yokohara/Kanagawaken, Japan). The animals were individually housed in a room maintained at $24 \pm 2{ }^{\circ} \mathrm{C}$ and $50 \pm 5 \%$ humidity with a $12 \mathrm{~h}$ light-dark cycle. They were fed on a commercial chow diet (Oriental Yeast Co. Ltd., Chiba, Japan) ad libitum and allowed free access to water. Eight groups of animals were studied at this time: (1) $15 \mathrm{mg} \mathrm{CAP} / \mathrm{kg}$, killed after exercise to exhaustion ( $n$ 6); (2) $15 \mathrm{mg} \mathrm{CAP} / \mathrm{kg}$, killed at rest (n 6); (3) $10 \mathrm{mg} \mathrm{CAP} / \mathrm{kg}$, killed after exercise to exhaustion (n 6); (4) $10 \mathrm{mg} \mathrm{CAP} / \mathrm{kg}$, killed at rest ( $n$ 6); (5) $6 \mathrm{mg} \mathrm{CAP/}$ $\mathrm{kg}$, killed after exercise to exhaustion $(n 6)$; (6) $6 \mathrm{mg}$ $\mathrm{CAP} / \mathrm{kg}$, killed at rest ( $n$ 6); (7) vehicle-treated, killed after exercise to exhaustion ( $n$ 6); (8) vehicle-treated, killed at rest $(n 7)$.

Two weeks before the experiment, all the rats were allowed to swim for $30 \mathrm{~min} / \mathrm{d}$ to accustom them to swimming and to being handled. Thereafter, rats were fasted overnight, and then both swimming and resting rats were given orally either vehicle or CAP at the doses of 6,10 or $15 \mathrm{mg} / \mathrm{kg}$ body weight, $2 \mathrm{~h}$ before exercise, by stomach intubations using a round-ended needle (Fig. 1). During the days of experimentation, rats allocated to groups 1, 3, 5 and 7 swam until exhaustion. Groups 2, 4, 6 and 8 rested in their cage for the corresponding time. Swimming exercise was carried out in a circular tank (diameter $0.50 \mathrm{~m}$, depth $0.55 \mathrm{~m}$ ) filled with water maintained at a temperature of $36 \pm 2{ }^{\circ} \mathrm{C}$. The water depth, $0.35 \mathrm{~m}$, was set so that the rats could not rest by supporting the tail on the bottom of the tank. Each of the rats had a weight attached ( $3 \%$ body weight) to the tail for the duration of the swim-to-exhaustion exercise. This exercise corresponds to an intensity of approximately four to five metabolic equivalents (Dawson \& Horvath, 1970). The animals were assessed to be exhausted when they failed to rise to the surface of the water to breathe within $7 \mathrm{~s}$. At this moment, the animals were removed from the tank. They were anaesthetized with sodium pentobarbitate and killed by exsanguination from the carotid artery before exercise or immediately after exhaustion. Blood plasma was collected and stored at $-80^{\circ} \mathrm{C}$ in a deep freeze for
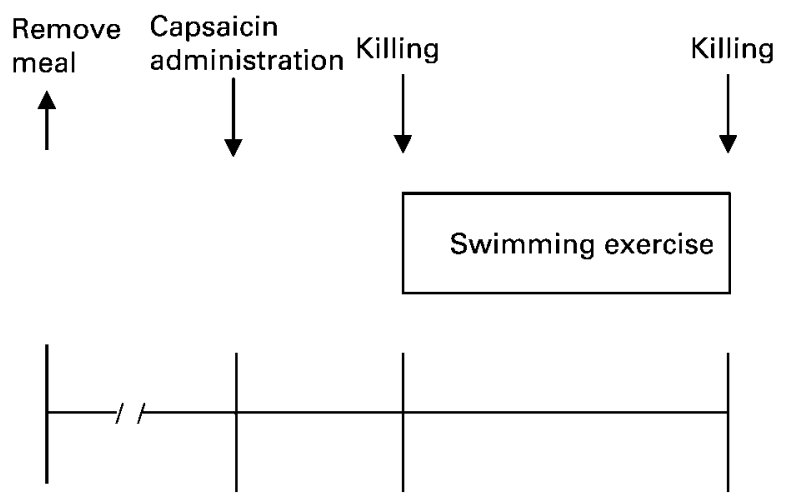

$-10 \mathrm{~h}$ $-120 \min$ 0

Exhaustion

Fig. 1. Protocol for swimming exercise in the rats. The swimming exercise was performed until exhaustion with a weight $(3 \%$ body weight) attached to the rat's tail. Animals were assessed to be fatigued when they failed to rise to the surface of the water to breathe within a $7 \mathrm{~s}$ period. For details of procedures, see pp. 515-516. future analysis for catecholamine, glucose, NEFA, insulin and glucagon concentrations with commercial kits (Wako Pure Chemical Industries, Chu-ku Osaka, Japan). Liver and muscles (white and red gastrocnemius) were removed and then frozen in liquid $\mathrm{N}_{2}$ to hold glycogen intact. Liver and muscle glycogen content was determined using the method of Lo et al. (1970). Briefly, portions of the muscle and liver were put in a tube containing $0.5 \mathrm{ml}$ $\mathrm{KOH}(300 \mathrm{ml} / \mathrm{l})$ saturated with $\mathrm{Na}_{2} \mathrm{SO}_{4}$ and immersed in a boiling water-bath for 20-30 min, before glycogen was assayed using a commercial kit (Glycogen Test Wako; Wako Pure Chemical Industries). To avoid circadian variations in physical activity, swimming was done from 10.00 to 17.00 hours, a period in which minimal variation of the endurance capacity has been confirmed in rats (Conlee et al. 1976).

\section{Statistical methods}

Results are expressed as mean values with their standard errors. The statistical significance $(P<0.05)$ of differences was determined by ANOVA followed by a Fisher Protected Least Significant Difference (PLSD) post hoc analysis.

\section{Results}

\section{Endurance swimming time}

The groups treated with $15 \mathrm{mg} \mathrm{CAP} / \mathrm{kg}$ showed a significant increase in swimming time to exhaustion of $219 \%$ compared with non-CAP-treated groups (156 (SE 38) min, $P<0.05)$. At the 6 and $10 \mathrm{mg} / \mathrm{kg}$ levels, no clear effect of CAP was observed (Fig. 2).

\section{Blood measurements}

Plasma adrenalin levels in both 10 and $15 \mathrm{mg}$ CAP/kg groups were significantly higher at exhaustion compared with that at rest $(P<0.05)$ (Fig. 3(a)). Plasma noradrenalin levels in all the groups treated with CAP were significantly higher at exhaustion compared with those at rest $(P<0.05)$. At exhaustion, plasma noradrenalin level in $15 \mathrm{mg} \mathrm{CAP} / \mathrm{kg}$ group was significantly $(P<0 \cdot 01)$ higher than that in nonCAP-treated group, though at rest, plasma noradrenalin level in $15 \mathrm{mg} \mathrm{CAP} / \mathrm{kg}$ group was significantly $(P<0.05)$ higher than non-CAP-treated group (Fig. 3(b)). In the rest groups, plasma NEFA was not significantly increased by CAP administration. However, at exhaustion, plasma NEFA was significantly higher in the $15 \mathrm{mg} / \mathrm{kg}$ group than in the non-CAP-treated group $(P<0.05)$ and it was significantly higher in all the groups treated with CAP at exhaustion than in the rest groups $(P<0.05)$ (Fig. 4(a)). Plasma glucose was not increased by CAP administration in the rest groups. However, at exhaustion, plasma glucose was significantly higher in the $15 \mathrm{mg} / \mathrm{kg}$ group than that in the non-CAP-treated counterpart $(P<0.05)$ and significantly lower than in the rest groups $(P<0.05)$ (Fig. 4(b)). The exercise until exhaustion caused a significant reduction of plasma insulin and an increment of glucagon in the all groups including the non-CAP-treated groups $(P<0 \cdot 05)$ (Fig. 5). 


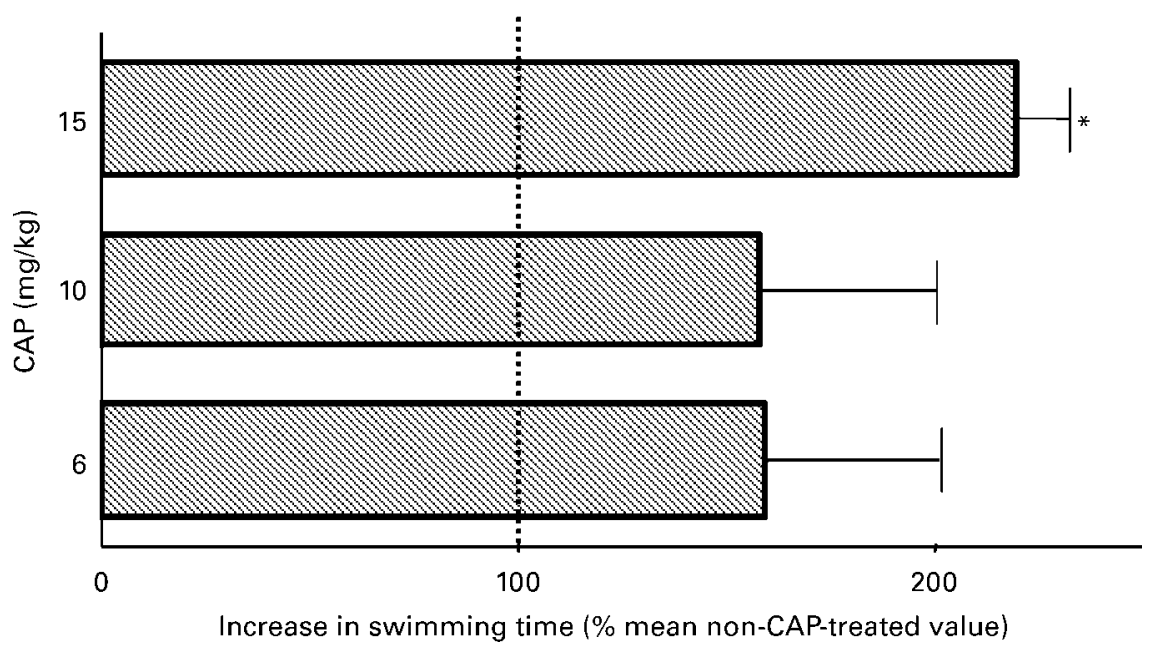

Fig. 2. Comparison of swimming times until exhaustion among three different doses of capsaicin (CAP). Values are means with their standard errors shown by bars ( $n$ 6-7 per group). For details of procedures, see pp. 515-516. Mean value was significantly different from that of the non-CAP-treated group: ${ }^{\star} P<0.05$.

\section{Glycogen contents}

Glycogen contents in liver and gastrocnemius muscles (red, white) were not affected by CAP $(15 \mathrm{mg} / \mathrm{kg})$ administration at rest, but both muscle glycogen contents were significantly higher in the CAP $(15 \mathrm{mg} / \mathrm{kg})$ group than in the non-CAP group at exhaustion $(P<0.05)$ (Table 1).

\section{Discussion}

CAP is well known to stimulate lipolysis and/or fat oxidation during exercise; the related hormonal and metabolic responses with enhanced lipolysis to its graded dosages have not yet been sufficiently investigated. In the present study, we examined the physiological responses to various dose levels of CAP in rats. The glycogen-sparing effect provides an important survival advantage for improving endurance performance time, because glycogen depletion is associated with physical exhaustion and slower utilization of glycogen results in improved endurance capacity.

The results of the present study showed that oral administration of $>15 \mathrm{mg} \mathrm{CAP} / \mathrm{kg}$ affects the glycogen content in the liver and muscle (white and red gastrocnemius) as well as plasma catecholamine, glucose, NEFA, insulin, glucagon at rest and swimming time to exhaustion. This conclusion is similar to that of Kim et al. (1997) following oral administration of $10 \mathrm{mg} \mathrm{CAP} / \mathrm{kg}$.

The results suggests that oral administration of $>15 \mathrm{mg}$ $\mathrm{CAP} / \mathrm{kg}$ is involved in the increase of the endurance
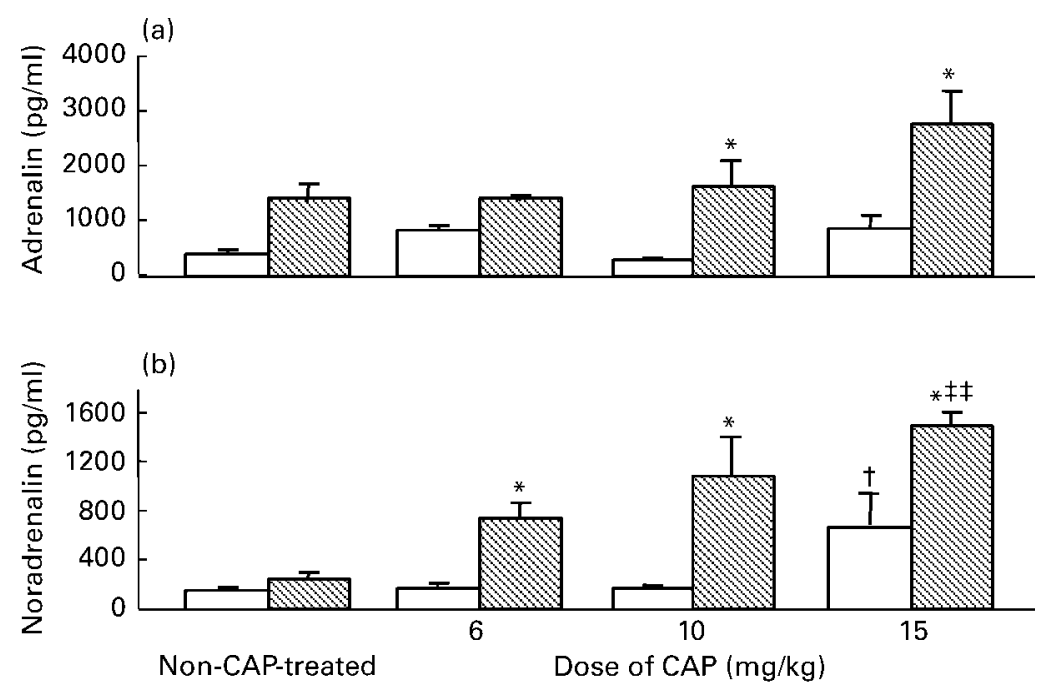

Fig. 3. Effects of various doses of capsaicin (CAP) and swimming to exhaustion on plasma adrenalin (a) and noradrenalin (b). $\square$, Rest; $\mathbb{Q}$, exhaustion. Values are means with their standard errors shown by vertical bars ( $n 6-7$ per group). For details of procedures, see pp. $515-$ 516. Mean values were significantly different from those of the same group at rest: ${ }^{\star} P<0.05$. Mean values were significantly different from those of the non-CAP-treated group: $† P<0.05$. Mean values were significantly different from those of the non-CAP-treated group after swimming to exhaustion: $\ddagger \ddagger P<0.01$. 
(a)
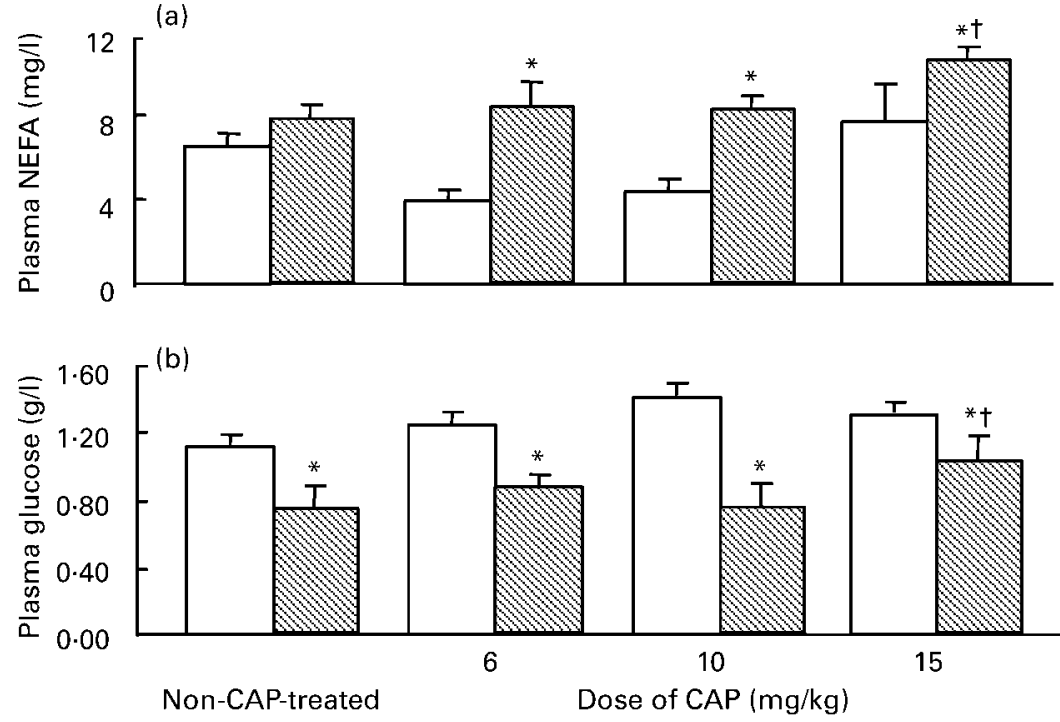

Fig. 4. Effects of various doses of capsaicin (CAP) and swimming to exhaustion on plasma non-esterified fatty acids (NEFA) (a) and glucose (b). $\square$, Rest; $\mathbb{\mathbb { N }}$, exhaustion. Values are means with their standard errors shown by vertical bars ( $n 6-7$ per group). For details of procedures, see pp. 515-516. Mean values were significantly different from those of the same group at rest: ${ }^{*} P<0.05$. Mean values were significantly different from those of the non-CAP-treated group: $† P<0.05$.

capacity after exercise to exhaustion. However, administration of only $10 \mathrm{mg} \mathrm{CAP} / \mathrm{kg}$ resulted in a higher muscle glycogen after swimming (Kim et al. 1997). It was thus concluded: 'The maximal effect was observed at a dose of $10 \mathrm{mg} / \mathrm{kg}$ while more than $15 \mathrm{mg} / \mathrm{kg}$ had no effect.' We disagree with this conclusion because they used a short time $(30 \mathrm{~min})$ and this was insufficient to create a significant increase in plasma catecholamine levels.

Winder (1986) reported that caffeine elevating the plasma concentration of NEFA decreased the rate of glycogen depletion during exercise and would result in boosting lipid oxidation and depressing muscle glycogen utilization. Fat oxidation is enhanced with increased NEFA concentration. Klein et al. (1993) reported that about half of the NEFA mobilized from adipose tissue was utilized in contractive muscles during moderate intensity endurance exercise. In the present study, a significant increase of NEFA was observed at various doses of CAP groups. These results suggest that the glycogen thus saved could become an available energy source for the following phases of exercise, which delays onset of fatigue.

Catecholamine is well known to play a crucial role in the prevention of hypoglycaemia during exercise (Wasserman et al. 1984). CAP significantly increased circulating catecholamine levels with successively improved endurance performance (Lim et al. 1997). Graham \& Spriet (1991) also found that CAP increased circulating catecholamine levels during exercise, but they did not observe the different dose effects of CAP on plasma catecholamine level at exhaustion. As shown in Fig. 3, the highest dose of CAP $(15 \mathrm{mg} / \mathrm{kg})$ increased plasma noradrenalin concentrations at exhaustion.
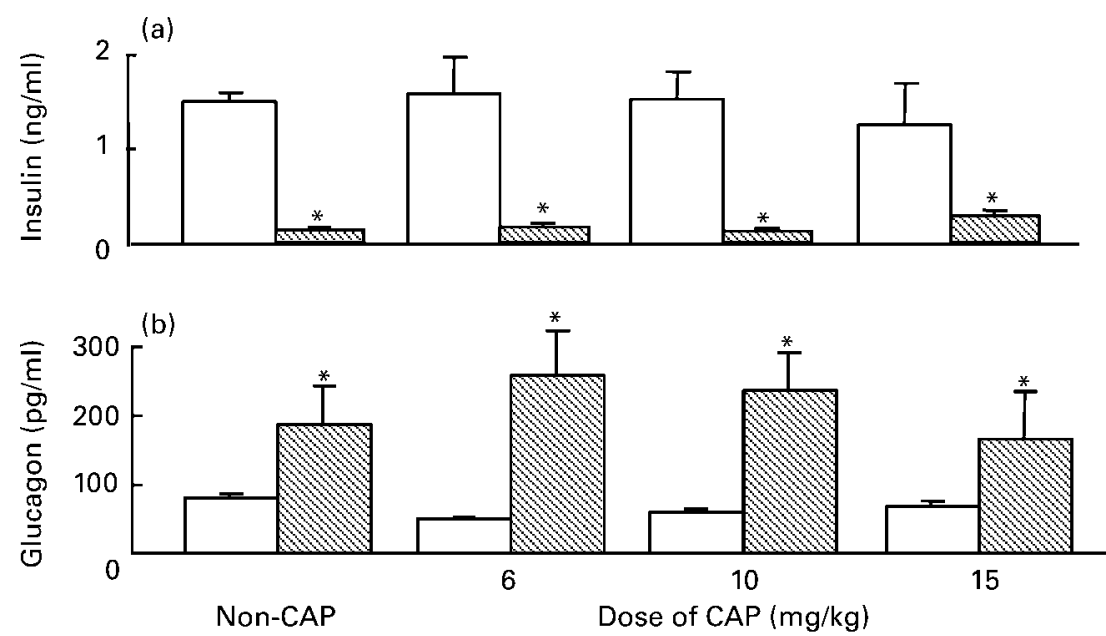

Fig. 5. Effects of various doses of capsaicin (CAP) and swimming to exhaustion on plasma insulin (a) and glucagon (b). $\square$, Rest; $\mathbb{Q}$, exhaustion. Values are means with their standard errors shown by vertical bars ( $n 6-7$ per group). For details of procedures, see pp. $515-516$. Mean values were significantly different from those of the same group at rest: ${ }^{*} P<0.05$. 
Table 1. Effect of $15 \mathrm{mg}$ capsaicin $/ \mathrm{kg}$ on liver and muscle glycogen $\ddagger$ (Mean values with their standard errors for four to seven rats per group)

\begin{tabular}{|c|c|c|c|c|}
\hline & \multicolumn{2}{|c|}{ Rest } & \multicolumn{2}{|c|}{ Exhaustion } \\
\hline & Mean & $\mathrm{SE}$ & Mean & SE \\
\hline \multicolumn{5}{|l|}{ Liver (mg/g) } \\
\hline Non-CAP & 55.4 & $5 \cdot 0$ & $32 \cdot 9^{\star \star}$ & $6 \cdot c$ \\
\hline CAP & $69 \cdot 7$ & $20 \cdot 0$ & $34 \cdot 1^{\star \star}$ & $10 \cdot c$ \\
\hline \multicolumn{5}{|c|}{ Gastrocnemius (mg/g) } \\
\hline \multicolumn{5}{|c|}{ White } \\
\hline Non-CAP & 4.04 & 0.2 & $0.79^{\star \star}$ & 0.3 \\
\hline CAP & 3.66 & 0.2 & $1.90^{* *} \dagger$ & 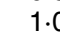 \\
\hline \multicolumn{5}{|l|}{ Red } \\
\hline Non-CAP & 4.02 & 0.3 & $1 \cdot 15^{\star \star}$ & 0.4 \\
\hline CAP & 3.87 & 0.1 & $1.52^{* *} \dagger$ & 0.5 \\
\hline
\end{tabular}

CAP, capsaicin.

Mean values were significantly different from those at rest for the same treatment group: ${ }^{\star \star} P<0.01$

Mean values were significantly different from those of the corresponding non-CAP-treated group: $\uparrow P<0.05$

$\ddagger$ For details of treatments and procedures, see pp. 515-516.

The metabolic effect of CAP on increasing endurance performance supported the notion of the sparing of muscle glycogen by oral administration of CAP $2 \mathrm{~h}$ before exercise (Kim et al. 1997). The glycogen saved becomes an available energy source for the following phase of exercise, which increase endurance capacity. Glycogen concentrations in muscle, as shown in Table 1, were reduced more slowly during exercise in CAP $(15 \mathrm{mg} / \mathrm{kg})$ group than in the non-CAP group. These results suggest that NEFA mobilized by CAP administration is used as a main energy source during exercise to exhaustion in rats, which enhances exercise time to exhaustion.

Costill et al. (1978) reported increased time to exhaustion with caffeine ingestion. Ivy et al. (1979) also reported enhanced exercise performance and found a significant difference in lipid oxidation. In the present study, endurance time to exhaustion was significantly higher in the highest dose CAP group compared with the non-CAP-treated group $(P<0.05)$. Decreased insulin secretion and increased glucagon secretion are involved in the precise matching of increased glucose production with increased glucose utilization that normally prevents hypoglycaemia during exercise (Issekutz \& Vranic, 1980; Wolfe et al. 1986; Wasserman et al. 1989a). As shown in Fig. 5, plasma insulin secretion was significantly higher $(P<0.05)$ in the group at rest compared with the exhaustion, but plasma glucagon secretion was significantly higher after exhaustion compared with resting.

In summary, $>15 \mathrm{mg} \mathrm{CAP} / \mathrm{kg} 2 \mathrm{~h}$ prior to exercise enhances endurance performance. We propose that the $>15 \mathrm{mg} \mathrm{CAP} / \mathrm{kg}$ is a lipolytic food for its significant effects on glycogen sparing for two reasons: first, $>15 \mathrm{mg}$ CAP/kg enhances lipolysis activity and this maintains higher NEFA concentrations during endurance exercise; second, glycogen spared by $>15 \mathrm{mg} \mathrm{CAP} / \mathrm{kg}$ can be used as an important energy source at the latter stage of exercise, especially when the exercise intensity is increased at that point. Therefore, a high dose of CAP is a useful ergogenic acid when given $2 \mathrm{~h}$ prior to endurance exercise in rats. Further work to elucidate the mechanism by which CAP promotes sparing of glycogen will be clearly warranted. The results presented here show that a high dose of CAP can dramatically alter many normal physiological and metabolic responses to endurance performance.

\section{Acknowledgement}

We thank the laboratory staff of the Nutrition Department of Waseda University for their advice and support.

\section{References}

Azevedo JL, Linderman JK, Lehman SL \& Brooks GA (1998) Training decreases muscle glycogen turnover during exercise. Eur J Appl Physiol 78, 479-486.

Conlee RK, Rennie MJ \& Winder WW (1976) Skeletal muscle glycogen content: diurnal variation and effects of fasting. Am J Physiol 231, 614-618.

Costill DL, Dalsky GP \& Fink WJ (1978) Effects of caffeine ingestion on metabolism and exercise performance. Med Sci Sports 10, 155-158.

Dawson CA \& Horvath SM (1970) Swimming in small laboratory animals. Med Sci Sports Exerc 2, 51-78.

Graham TE \& Spriet LL (1991) Performance and metabolic responses to a high caffeine dose during prolonged exercise. J Appl Physiol 71, 2292-2298.

Issekutz B \& Vranic M (1980) Role of glucagons in regulation of glucose production in exercising dogs. Am $J$ Physiol 238, E13-E20.

Ivy JL, Costill DL, Fink WJ \& Lower RW (1979) Influence of caffeine and carbohydrate feeding on endurance performance. Med Sci Sports 11, 6-11.

Kawada T, Watanabe T, Takaishi T, Tanaka T \& Iwai K (1986) Capsaicin-induced beta-adrenergic action on energy metabolism in rats: influence of capsaicin on oxygen consumption, the respiratory quotient, and substrate utilization. Exp Biol Med 183, 250-256.

Kim KM, Kawada T, Ishihara K, Inoue K \& Fushiki T (1997) Increase in swimming endurance capacity of mice by capsaicin-induced adrenal catecholamine secretion. Biosci Biotechnol Biochem 61, 1718-1723.

Klein S, Coyle EF \& Wolfe RR (1993) Fat metabolism during low-intensity exercise in endurance-trained and untrained men. Am J Physiol 267, E934-E940.

Leighton B \& Foot EA (1995) The role of the sensory peptide calcitonin-gene-related peptide(s) in skeletal muscle carbohydrate metabolism: effects of capsaicin and resiniferatoxin. Biochem J 307, 707-712.

Lim K, Yoshioka M, Kikuzato S, et al. (1997) Dietary red pepper ingestion increases carbohydrate oxidation at rest and during exercise in runners. Med Sci Sports Exerc 29, 355-361.

Lo SJ, Russell C \& Taylor AW (1970) Determination of glycogen in small tissue samples. J Appl Physiol 28, 234-236.

Matsuo T, Yoshioka M \& Suzuki M (1996) Capsaicin in diet does not affect glycogen contents in the liver and skeletal muscle of rats before and after exercise. J Nutr Sci Vitaminol (Tokyo) 42, $249-256$.

Oh TW (2001) Effects of capsaicin administration on brown adipose tissue oxygen consumption in Wistar-Kyoto and spontaneously hypertensive rat. Hum Sci Res 10, 229-239.

Wachtel RE (1999) Capsaicin. Reg Anesth Pain Med 24, 361-363.

Wasserman DH, Lavina H, Lickley A \& Vranic M (1984) Interactions between glucagons and other counterregulatory 
hormones during normoglycemic and hypoglycemic exercise in dogs. J Clin Invest 74, 1404-1473.

Wasserman DH, Spalding JA, Lacy DB, Colburn CA, Goldstein RE \& Cherrington AD (1989a) Glucagon is a primary controller of hepatic glycogenolysis and gluconeogenesis during muscular work. Am J Physiol 257, E108-E117.

Wasserman DH, Williams PE, Lacy DB, Goldstein RE \& Cherrington AD (1989b) Exercise-induced fall in insulin and hepatic carbohydrate metabolism during muscular work. Am $J$ Physiol 256, E500-E509.

Winder WW (1986) Effect of intravenous caffeine on liver glycogenolysis during prolonged exercise. Med Sci Sports Exerc 18, 192-196.

Wolfe RR, Nadel ER, Shaw JHF, Stephenson LA \& Wolfe MH (1986) Role of changes in insulin and glucagons in glucose homeostasis in exercise. J Clin Invest 77, 900-907. 